\title{
A novel variable rate pneumatic fertilizer applicator
}

\begin{abstract}
The canopy prevents the use of global positioning system based fertilizer applicators in oil palm plantations. Hence, a radio frequency identification triggered variable rate pneumatic fertilizer system was developed for this application. A real-time embedded system was used as the core controller, LabVIEW software was used to program and coordinate the operations of the embedded system and the host computer. A speed measuring unit was used to provide feedback to the system. A field test was conducted to examine the response time. The sensors were calibrated in the laboratory and the measurement linearity had regression coefficients close to 1. Two to three seconds were required for the device to respond to changes in application rate. It is expected that this approach will become an alternative in plantations where the canopy hinders proper application of global positioning systems.
\end{abstract}

Keyword: Fertilizer application; Precision farming; Radio frequency identification; RFID; Variable rate technology 